\title{
Modeling and Simulation of Liquid Propellant Rocket Engine Transient Performance Using Modelica
}

\author{
Liu Wei ${ }^{1}$ Chen Liping ${ }^{1}$ Xie Gang ${ }^{1}$ Ding $\mathrm{Ji}^{2}$ Zhang Haiming ${ }^{2}$ Yang Hao ${ }^{2}$ \\ ${ }^{1}$ School of Mechanical Science \& Engineering, Huazhong University of Sci. \& Tech., China, \\ liuwei20@foxmail.com, \{chenlp, xieg\} @tongyuan.cc \\ ${ }^{2}$ Suzhou Tongyuan Software \& Control Technology Co., Suzhou, China, \\ \{dingj, zhanghm, yangh \} \& tongyuan. cc
}

\begin{abstract}
This paper presents a liquid propellant rocket engine (LPRE) model library in Modelica language, which contains component models such as pipes, valves, tanks, turbo-pumps, combustion chambers, nozzles, injectors, gas generators, etc. These component models can be applied to establish a variety of liquid rocket engine systems with the capability of predicting engine transient performance during the startup, shutdown and regulation processes. Typical gas-pressurized liquid propellant engine system and turbo-pump liquid propellant engine system are modeled in the paper. Some simulations and analyses are performed to validate the models qualitatively. All the modeling and simulations are implemented in MWorks (Zhou, 2006), which is a modeling and simulation platform that fully supports Modelica.
\end{abstract}

Keywords: $\quad$ liquid propellant engine, thermo-fluid, startup and shutdown transient

\section{Introduction}

Liquid propellant rocket engines are widely used and play a very important role in aerospace. The function of a LPRE is to generate thrust through chemical reactions, which usually release thermal energy from the chemical energy of the propellants. The pressure generated from the thermal energy imparts a momentum to the reaction products. Then a momentum in the opposite direction is imparted to the rocket and propels a vehicle in space. A LPRE system usually consists of thrust chamber assembly, propellant feed system, turbine-drive system (for turbo-pump LPRE), and propellant control system, etc. A liquid propellant rocket engine is very complex and difficult to design and analyze because of many coupled subsystems and their extreme working conditions. Physical experiments under various conditions are also expensive. Hence it's critical to utilize models to facilitate the design and analysis process of LPRE. The control equations of LPRE dynamic motion are implicit and nonlinear differential algebraic equations. The structure and components of one LPRE often differ from those of another. Therefore, it is a big challenge for engineers to build LPRE models that are of high generality and reusability, flexibility. Most of the existing models for LPRE (Karimi, 2003; Ruth, 1990; Matteo, 2012; Tabrizi, 2013; Karimi, 2006) lack generality, reusability or flexibility. A usual completed system model can only be applied to some specific LPRE and cannot be modified directly to be applied to others. Besides, the modeling process is difficult and time consuming, because engineers have to consider all of the numerical problems in equations solving procedure.

Modelica (Fritzson, 2010) is an object-oriented equation-based modeling language, which is capable of multi-domain modeling and has a strong software component model with structure for creating and connecting components. Modelica allows engineers to use mathematics to define system behaviors naturally and have powerful structuring capability to deal with complex interconnected systems. Engineers often do not need to consider numerical solving problem, thanks to the Modelica developing environment. These properties make Modelica suitable for the modeling and simulation problems of large scale and complex LPRE system. This paper focuses on modeling general library and efficient simulation of LPRE using Modelica.

\section{Implementation of component models}

We try to make best use of capabilities of Modelica when developing the liquid propellant rocket engine library. Firstly, we determine the objective of the library. A system model is expected to predict the flow rate, pressure and temperature of the components in a LPRE system during the whole running time. The dynamics of LPRE mainly consists of fluid dynamics, heat transfer, thermal dynamics and combustion, all of which should be taken into consideration.

According to the natural border in the LPRE system and object orientation, we divide a system into interacting components. Object orientation is viewed as a structuring tool to handle the topological structure description of a LPRE system. In order to decrease 
complexity, we assume that the decomposed components are independent physical functional objects, including pipes, valves, tanks, turbo-pumps, combustion chambers, nozzles, injectors, gas generators and bottles. While subject decomposition (Jensen, 2003) is also performed to obtain base models, which collect common properties of a class of models and are physical phenomenon units. The main base models in LPRE library consist of control volume, flow model, ideal gas property, heat convection, heat conduction and combustion model. These base models cannot be simulated directly, and are inherited and aggregated by more than one component model for reusing.

The components models exchange information through connectors. The connectors ensure that components are independent of each other and work under a set of boundary conditions provided by connectors. The connectors of component models should present the properties of interactions between these components in a real physical LPRE system. Thus it would be easy and natural to connect components. The LPRE library contains four connectors for fluid flow, heat flow, 2D rotation and 2D translation respectively. In order to make the LPRE library consistent with Modelica Standard Library (MSL) and increase versatility, the four kinds of the connectors in the LPRE library are the same with those in MSL. Using base models, connectors and mathematical models of physical components, detailed component models are developed and implemented.

\section{Description of mathematical models}

In this chapter, the mathematical models of some most important components are presented, including combustion chamber, nozzle, pipe, valve, pump and turbine.

\subsection{Combustion chamber}

The thrust chamber is a key subsystem of a LPRE. The combustion chamber is a part of the thrust chamber where the chemical reaction of the propellant takes place to generate hot gas products. It is assumed that liquid propellants react and change to hot gas after a constant delay time and gas flow in combustion chamber is adiabatic. The control equations of a bipropellant combustion chamber are described as follows.

$$
\begin{gathered}
\frac{d m_{o x}}{d t}=\dot{m}_{o x i}-\frac{m_{o x}}{\tau} \\
\frac{d m_{f u}}{d t}=\dot{m}_{f u i}-\frac{m_{f u}}{\tau} \\
\frac{d m_{g}}{d t}=\frac{m_{o x}+m_{f u}}{\tau}-\dot{m}_{g o}
\end{gathered}
$$

$$
\begin{gathered}
\frac{d K_{m}}{d t}=\frac{1+K_{m}}{m_{g}}\left(\frac{m_{o x}-m_{f u}}{\tau}\right) \\
\frac{d p}{d t}=\frac{R T}{V} \frac{d m_{g}}{d t}+\frac{p}{R T} \frac{d(R T)}{d t}+\frac{p}{V} \frac{d V}{d t} \\
\tau_{g} \frac{d R T}{d t}=R T_{i}\left(p, K_{m}\right)-R T
\end{gathered}
$$

Here $m_{o x}$ is the oxidizer mass, $m_{f u}$ is the fuel mass, $\dot{m}_{o x i}$ and $\dot{m}_{f u i}$ are the inlet mass flow rates of oxidizer and fuel, $\dot{m}_{g o}$ is the outlet mass flow rate of gas products, $K_{m}$ is the propellants mixture ratio, $p$ is the pressure inside chamber, $R T$ is the product of gas constant and temperature of gas staying in combustion chamber, $R T_{i}\left(p, K_{m}\right)$ is the product of gas constant and temperature of gas products defined as a function of pressure and mixture ratio, $\tau_{g}$ is the stay time of gas in chamber, $V$ is the volume of chamber. The control equations of a gas generator resemble those of the combustion chamber.

\subsection{Nozzle}

The nozzle is also a part of a thrust chamber, whose function is to accelerate gases and create high exhaust velocity. It is assumed that the gas flow through the nozzle is an isentropic expansion. Nozzle expansion ratio is defined as follows:

$$
\varepsilon=\frac{A_{e}}{A_{t}}=\frac{\left(\frac{2}{\gamma+1}\right)^{\frac{1}{\gamma-1}} \frac{p_{c}}{p_{e}}}{\sqrt{\frac{\gamma+1}{\gamma-1}\left[1-\left(\frac{p_{e}}{p_{c}}\right)^{\frac{\gamma}{\gamma-1}}\right]}}
$$

Here, $A_{e}$ and $A_{t}$ are the flow areas at nozzle exit and throat, $p_{c}$ and $p_{e}$ are the pressure at chamber and nozzle exit, $\gamma$ is the specific heat ratio.

Velocity at nozzle exit is given by:

$$
v_{e}=\sqrt{\frac{2 g \gamma}{\gamma-1} R T_{c}\left[1-\left(\frac{p_{e}}{p_{c}}\right)^{\frac{\gamma-1}{\gamma}}\right]}
$$

Here, $R$ is the gas constant, $T_{c}$ is gas temperature in combustion chamber.

The mass flow through a nozzle is given by:

$$
\dot{m}=\frac{A_{t} p_{c} \gamma}{\sqrt{\gamma R T_{c}}} \sqrt{\left(\frac{2}{\gamma+1}\right)^{\frac{\gamma+1}{\gamma-1}}}
$$

The thrust force is defined as follows:

$$
F=\dot{m} v_{e}+A_{e}\left(p_{e}-p_{a}\right)
$$

\subsection{Pipe}

Pipes are interconnect components that carry fluid to the intended components. Pressure drop between inlet 
and outlet of a pipe is determined by the following equation.

$$
\Delta p=\lambda \frac{l}{d} \rho \frac{v^{2}}{2}+\xi \rho \frac{v^{2}}{2}
$$

Here $\lambda$ is the friction coefficient, $\xi$ is the coefficient of the local head loss, $l$ and $d$ are length and diameter of the pipe, $\rho$ and $v$ are density and velocity of fluid in the pipe.

\subsection{Valve}

Valves control fluid flows. Every LPRE uses some of them. Liquid valves are governed by the following familiar equation, where the flow rate is the function of pressure drop and flow area:

$$
\dot{m}=C_{d} A \sqrt{2 \rho \triangle p}
$$

Here $C_{d}$ is the flow rate factor.

For a gas valve, when $\frac{p_{o}}{p_{i}} \geq\left(\frac{2}{\gamma+1}\right)^{\frac{\gamma}{\gamma-1}}$, we have

$$
\dot{m}=C_{d} A \sqrt{\frac{2 \gamma p_{i} \rho_{i}}{\gamma-1}\left[\left(\frac{p_{o}}{p_{i}}\right)^{\frac{2}{\gamma}}-\left(\frac{p_{o}}{p_{i}}\right)^{\frac{\gamma+1}{\gamma}}\right]}
$$

$$
\begin{aligned}
\text { When } \frac{p_{o}}{p_{i}}<\left(\frac{2}{\gamma+1}\right)^{\frac{\gamma}{\gamma-1}}, \text { then } \\
\dot{m}=C_{d} A \sqrt{\frac{2 \gamma p_{i} \rho_{i}}{\gamma+1}\left(\frac{2}{\gamma+1}\right)^{\frac{2}{\gamma-1}}}
\end{aligned}
$$

\subsection{Pump}

A pump pressurizes propellants and deliver them to extended components in a turbo-pump propellant feed system. Centrifugal pump is the most widely used pump type. Performance maps for head and power are used in the pump model. The head $h$ is evaluated by following equations:

$$
\begin{gathered}
h=\left(\frac{n}{n_{r e f}}\right)^{2} h_{q}\left(q \frac{n_{r e f}}{n}\right) \\
P=\left(\frac{n}{n_{r e f}}\right)^{3} \frac{\rho}{\rho_{r e f}} P_{q}\left(q \frac{n_{r e f}}{n}\right) \\
P=\omega \tau \\
\eta_{p}=\frac{\Delta p q}{P}
\end{gathered}
$$

Here, $n$ is the rotational speed, $q$ is the volume flow rate, $P$ is the power consumption, $\omega$ is the angular velocity, $\tau$ is the torque, $\eta_{p}$ is the pump efficiency, $h_{q}()$ and $P_{q}()$ are functions obtained from the performance map, ref is the reference value.

\subsection{Turbine}

A turbine gets energy from the expansion of high temperature and high pressure gas, and provides power to the pump. The expansion is assumed to be isentropic. The control equations of a turbine is presented as follows:

$$
\begin{gathered}
W=\frac{\gamma}{\gamma-1} R T_{i}\left[1-\left(\frac{p_{o}}{p_{i}}\right)^{\frac{\gamma-1}{\gamma}}\right] \\
\eta_{t}=\eta_{t}\left(\frac{u}{C}\right)=\frac{\omega \tau}{W \dot{m}}
\end{gathered}
$$

Here, $W$ is the power of gas expansion, $\eta_{t}\left(\frac{u}{c}\right)$ is the turbine efficiency defined as a function of the velocity ratio obtained from the performance map. The mass flow rate in a turbine is evaluated by the familiar equation in the gas valve model.

\section{Simulation and analysis of system}

Liquid propellant rocket engines area classified into two major types according to their propellant feed system, namely gas-pressurized liquid propellant rocket engine and turbo-pump liquid propellant rocket engine. Typically, engines with small propellant quantities have a gas-pressurized propellant feed system, and large engines required weight considerations choose a turbo-pump propellant feed system. The startup and shutdown phases of a LPRE are very complex. The engine components are working under extreme operating conditions, and about half of the engine failures occur during the startup and shutdown. Thus the prediction of the transient characteristics of a LPRE is important and necessary to engine safety and reliability. In the next sections, we model two typical kinds of the LPRE, and perform simulations to obtain the transient characteristics of the LPRE.

\subsection{Gas-pressurized liquid propellant rocket engine}

A gas-pressurized LPRE consists of gas bottles, propellant tanks, pipes, valves, thrust chamber heads, injectors, combustion chambers, nozzles and igniters. According to the typical physical structure of gaspressurized LPRE, a system model is quickly built by using component models in the LPRE model library. Figure 1 depicts the diagram view of the gaspressurized LPRE system model. Figure 2 shows the combustion chamber pressure. Two thrust chambers start up and shut down at different times. There is a pressure pulse during the start-up, because the oxidant and the fuel flow into the combustion chamber asynchronously. Thus it is important to control the difference between the times when two propellants flow into combustion chamber initially, in order to 
decrease the maximum of the pressure pulse. When the shut-down signal is given, the propellant control valves are closed. Pressure drops immediately, because the combustion lacks fuel and oxidant. The result also implies that the operations of one thrust chamber directly influence the steady pressure of another.

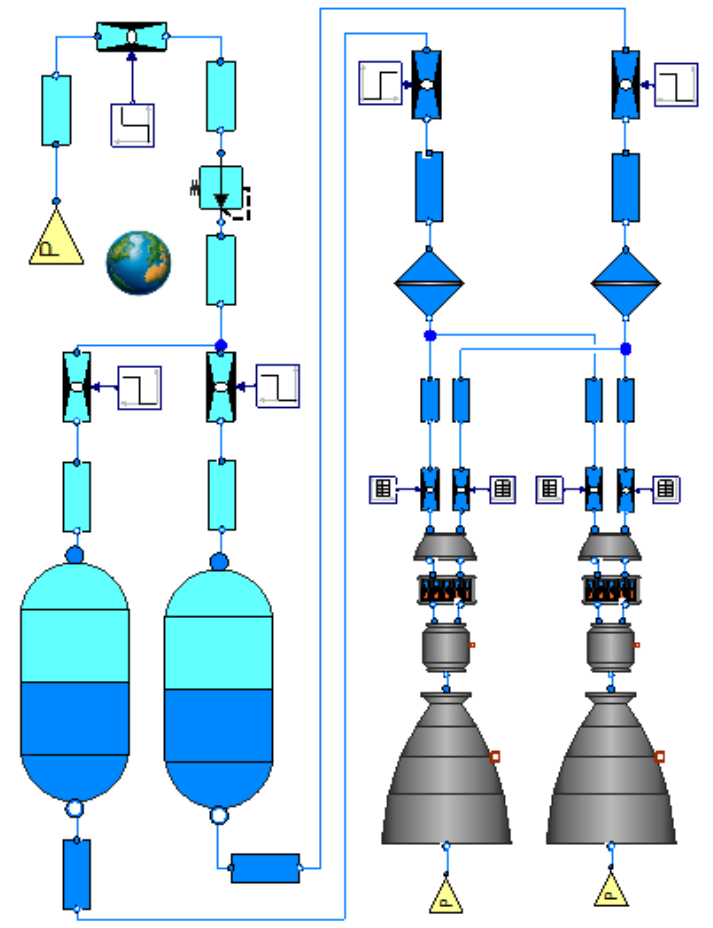

Figure 1. A gas-pressurized LPRE system Model

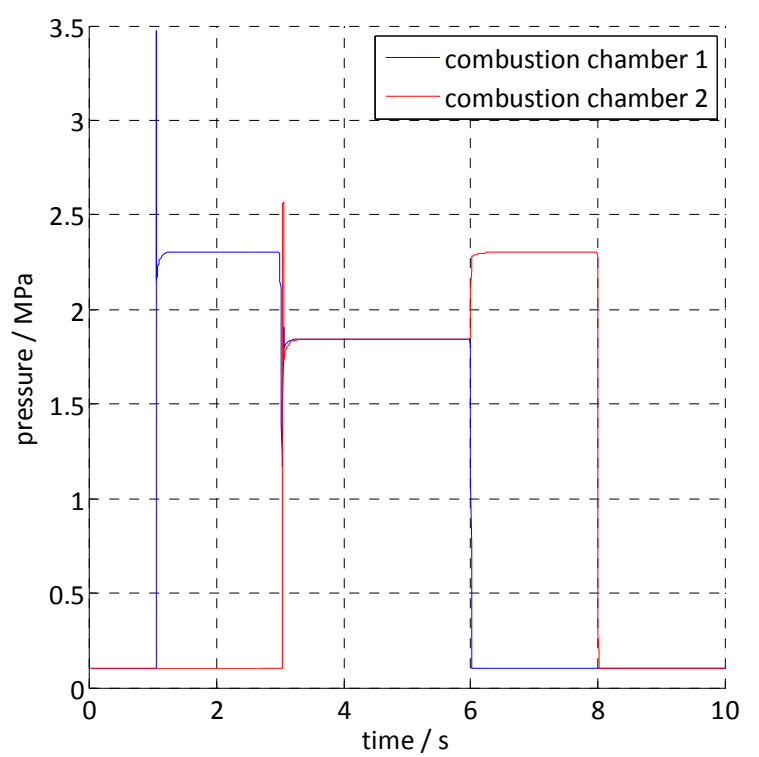

Figure 2. Pressure in the combustion chambers

\subsection{Turbo-pump liquid propellant rocket engine}

In contrast to the gas-pressurized LPRE, the turbopump LPRE has Turbo-pumps and a gas generator, but has no gas bottles. Figure 3 depicts the diagram view of a turbo-pump LPRE system model.

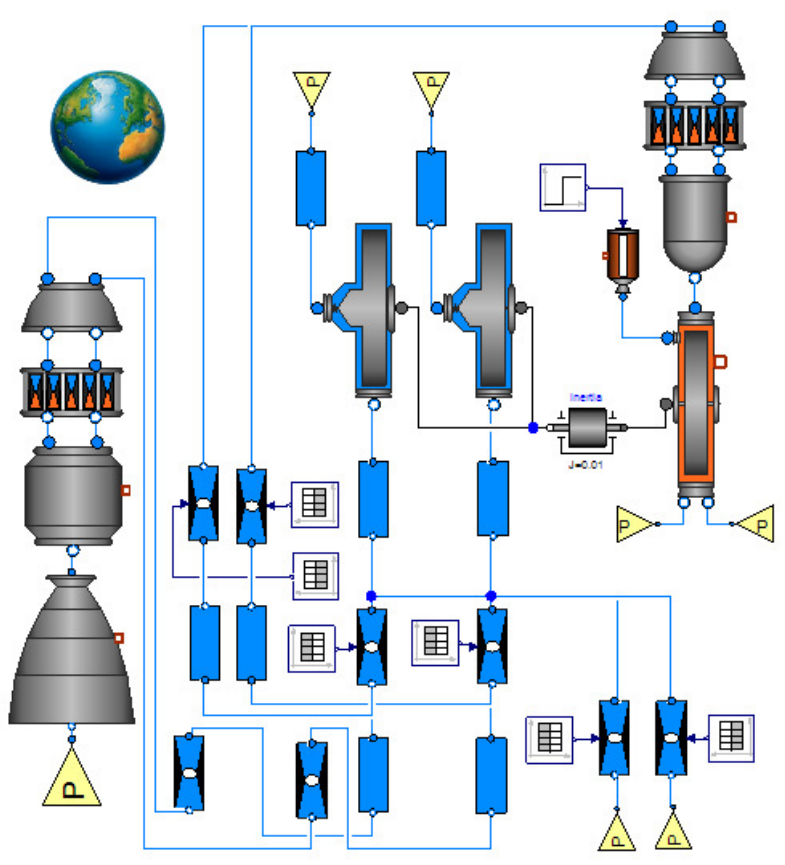

Figure 3. A turbo-pump LPRE system Model

Figures 4,5 and 6 show the turbine shaft speed, pump outlet pressure, combustion chamber pressure and gas generator pressure during the start-up. Firstly, the igniter drives the turbine to run and the turbine rational speed rises very quickly. The pump outlet pressure, as the function of the turbine rational speed, also increases. The pumps then deliver propellants to the combustion chamber and gas generator. After the gas generator is ignited, it drives the turbo-pump in turn. Due to couple relations between gas generator and turbo-pump, rational speed and pressure exceed the nominal ones, and then decrease and stabilize to the steady states.

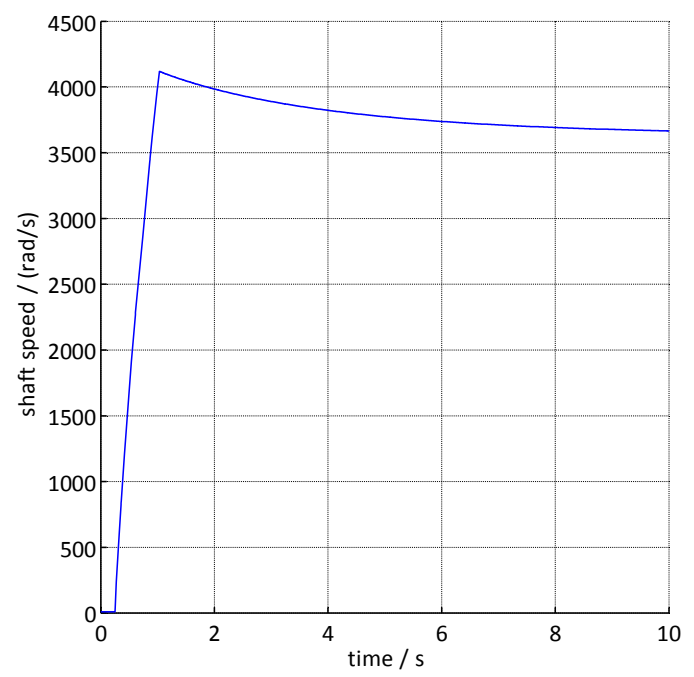

Figure 4. Rotational speed of turbine 


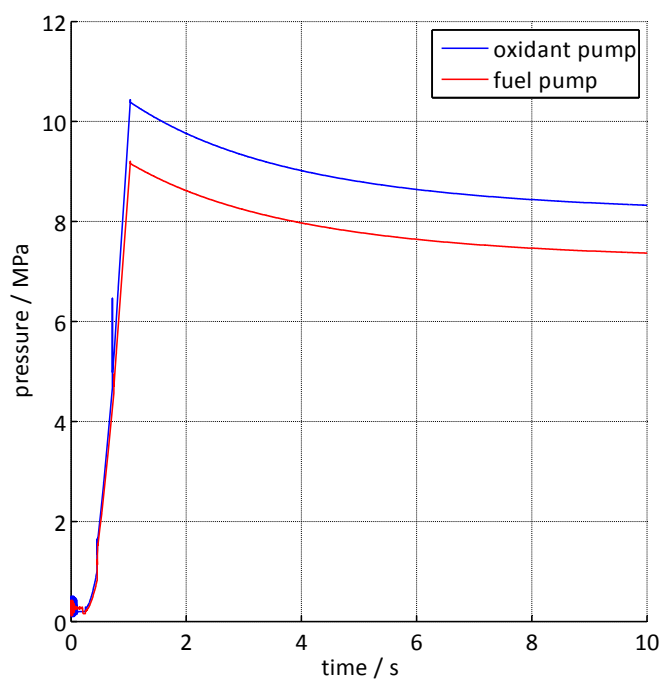

Figure 5. Pump outlet pressure

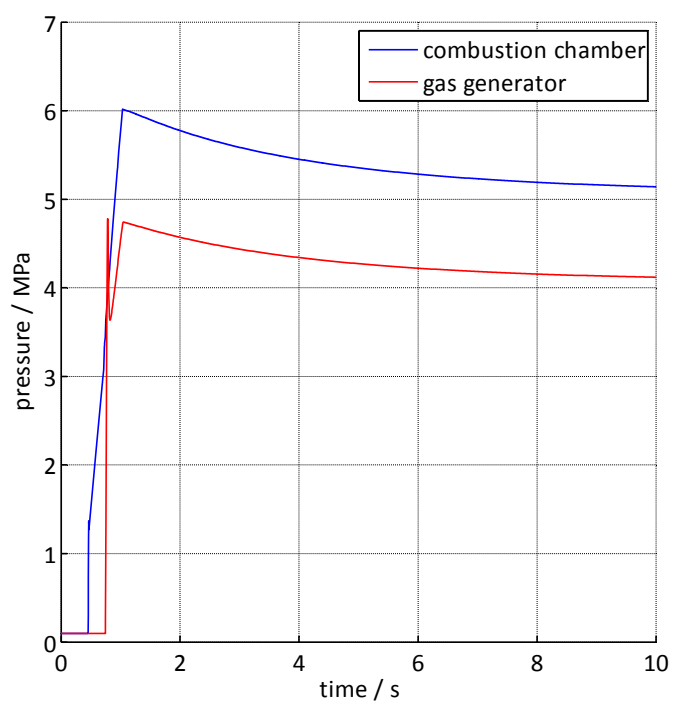

Figure 6. Pressure in combustion chamber and gas generator

Some interesting features of the shut-down process are depicted in Figures 7, 8 and 9. When the shut-down signal is given, the propellant control valves are closed. The gas generator lacks of propellants very soon, so the pressure in it begins to drop quickly. The rotational speed of turbine shaft decreases slowly, because the power delivered by gas generator to drive turbine get smaller and there exists resistance. There are residual propellants in the pipelines, thus pressure oscillations in the combustion chamber and gas generator are observed.

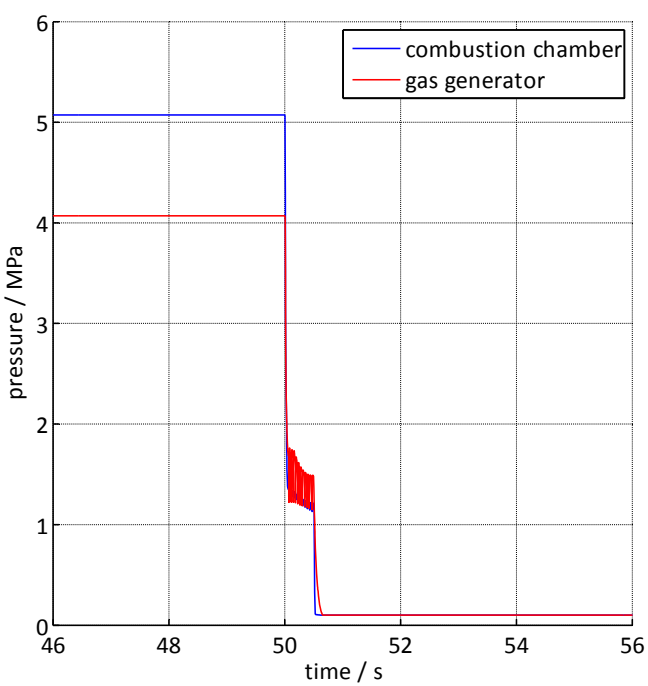

Figure 7. Pressure in combustion chamber and gas generator

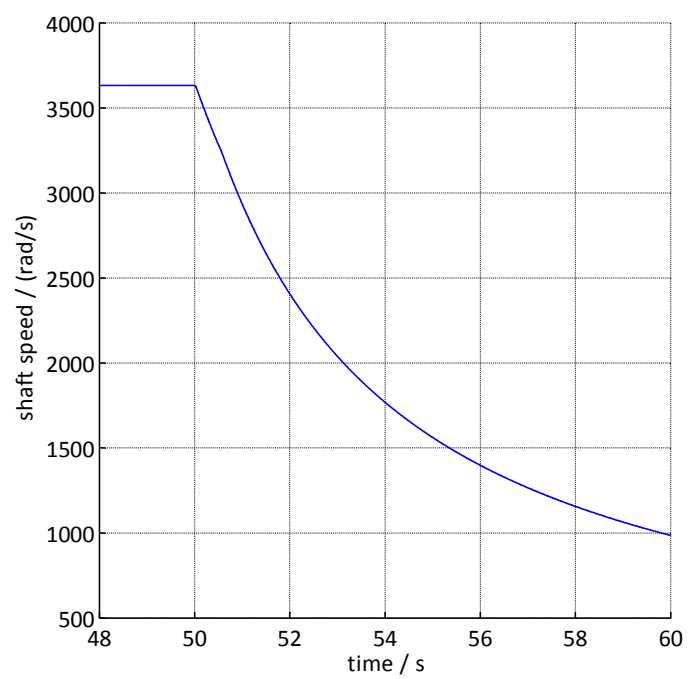

Figure 8. Rotational speed of turbine

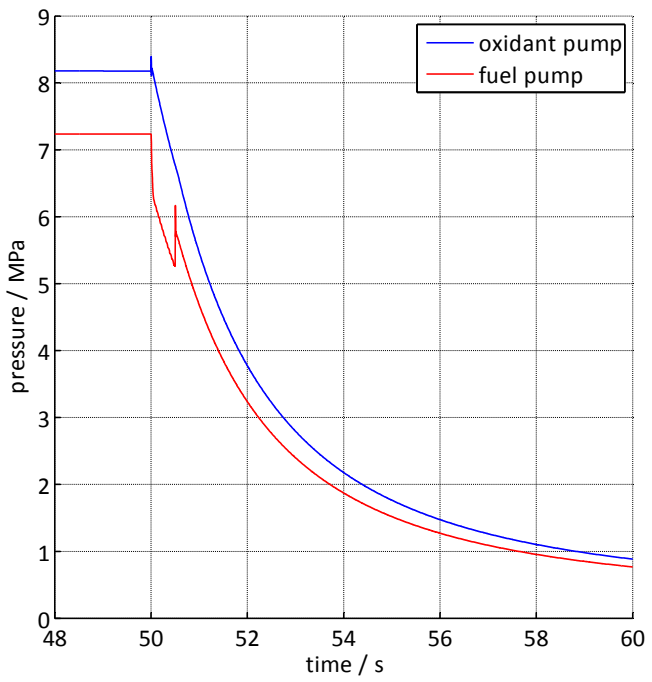

Figure 9. Pump outlet pressure 


\section{Conclusions}

We have established a component model library for liquid propellant rocket engine that can be used to build LPRE system models efficiently and simulate engine transient performance. In this paper, we give the control equations of some most interesting components of LPRE. The general method for applying the characteristics of Modelica, especially objectorientation and connection mechanism, to the modeling procedure of LPRE is presented. Gas pressurized LPRE and turbo-pump LPRE system models are build using component models from the established LPRE library. The transients during engine start-up and shutdown are simulated and analyzed. Due to extreme working conditions and uncertainty, the start-up and shut-down processes are very complex. Our LPRE library provides an efficient tool to study the transient properties. In the future, we will validate the system model with existing experimental results and improve accuracy.

\section{Acknowledgements}

The paper is supported by the Key Project of National High Technology Research and Development Program (No. 2013AA041301).

\section{References}

Fanli Zhou, Liping Chen, Yizhong Wu and et al. MWorks: a modern IDE for modeling and simulation of multi-domain physical systems based on Modelica. Proceedings of the 5th International Modelica Conference, Vol. 2: 725-731, 2006.

Peter Fritzson. Principles of object-oriented modeling and simulation with Modelica 2.1. John Wiley \& Sons, 2010.

H. Karimi, A. Nassirharand, and M. Behesht. Dynamic and nonlinear simulation of liquid-propellant engines. Journal of propulsion and power, 19(5): 938-944, 2003.

E. K. Ruth and R. L. Ahn. Advanced Liquid Rocket Engine Transient Model. 1990. AIAA-90-2299.

Francesco Di Matteo, Marco De Rosa, and Marcello Onofri. Transient Simulation of the RL-10A-3-3A Rocket Engine. Space Propulsion Conference. 2012.

Mahyar Naderi Tabrizi, Seyed Ali Reza Jalali Chime, and Hassan Karimi. Modeling and Simulation of Open Cycle Liquid Propellant Engines. Journal of Science and Engineering, 1(1): 17-34, 2013.

H. Karimi, and A. Nassirharand. Application of a Simulation Algorithm for Dynamic analysis of a Liquid Propellant Engine. Journal of Aerospace Science and Technology, 3(1): 23-30, 2006.

Jakob Munch Jensen. Dynamic Modeling of ThermoFluid Systems. Diss. Ph. D. thesis, Technical University of Denmark, 2003. 